Francois Rigaux

\title{
Multinationale Unternehmen, Staat und Recht
}

Vorbemerkung der Redaktion: Das „Ständige Tribunal der Völker“ - kein „offizieller“ Gerichtshof, sondern eine von der Basso-Stiftung getragene Institution (in Deutschland besser bekannt als „Basso-Tribunal“") - bereitet eine öffentliche Untersuchung zur Rolle der Multinationalen Konzerne in der Weltwirtschaft vor. In diesem Zusammenhang wurden auch erhebliche Vorwürfe gegen den französischen Konzern Elf-Aquitaine erhoben. In dem folgenden für die Arbeit des Tribunals verfaßten Text wird die Anklage gegen Elf zum Anlaß genommen, um die grundsätzlichen Probleme zu diskutieren, die einer juristischen Verfolgung multinationaler Konzerne entgegenstehen.

\section{Die Anklage}

Wenn ich die am 14. Januar 1999 von dem Kollektiv „Elf darf nicht die Gesetze in Afrika machen" erhobene Anklage recht verstehe, so werden zwei Angeklagte vor das Ständige Tribunal der Völker zitiert, die Firma Elf-Aquitaine und die Französische Republik. Der Generalsekretär des Tribunals hat daher zu Recht - im Einklang mit den Statuten des Tribunals den Vorstand von Elf sowie den französischen Ministerpräsidenten aufgefordert, die Argumente zu ihrer Verteidigung vorzutragen. Ich will nicht näher auf die Sachverhalte eingehen, die der Anrufung des Tribunals zugrunde liegen; ich möchte mich vielmehr auf die Erörterung einiger grundsätzlicher Probleme konzentrieren. Müssen die transnationalen Unternehmen die Grundrechte und das Völkerrecht in ihren Beziehungen zu den auswärtigen Staaten beachten? Haftet ein Staat, in dessen Machtbereich ein derartiges Unternehmen angesiedelt ist, für die Machenschaften dieses Unternehmens außerhalb des Staatsgebietes, in dem es seinen Sitz hat und nach dessen Recht es organisiert ist? Gibt es Vorschriften des Internationalen Rechts, die auf diese Sachverhalte anzuwenden sind? Welches sind die Rechtslehren, deren Anwendung die Straffreiheit der beiden Akteure ermöglicht und die notwendigerweise bekämpft und kritisiert werden müssen - was mir angesichts der Umstände eine der Aufgaben zu sein scheint, die uns gestellt worden ist. 


\section{Die Trennung von Öffentlichem und Privatem Recht}

\section{Ein Rückblick auf die klassische Rechtslehre}

Die französische Rechtslehre geht nach wie vor von der Aufspaltung des Rechts in zwei Hauptgebiete aus, dem Privatrecht und dem Öffentlichen Recht. Diese Auffassung findet ihren deutlichsten, wenn auch schon etwas veralteten Ausdruck im Werk von Carbonnier:

„Das gesamte Recht wird in zwei Teile gegliedert: Öffentliches Recht und Privatrecht. Das Öffentliche Recht befaßt sich mit der Organisation des Staates und seinen Beziehungen mit den Bürgern... Gegenstand des Privaten Rechts sind die Beziehungen von Privatleuten untereinander.“ (Carbonnier 19969, No. 11)

Der tautologische Charakter der Definition des Privatrechts springt ins Auge: die Beziehungen der Privatleute untereinander. Was soll das heißen? Geht ein multinationales Unternehmen privatrechtliche Beziehungen mit seinen Mitarbeitern ein, mit seinen Vertragspartnern, mit dem Staat, auf dessen Territorium es agiert, oder gar mit dem Staat, in dem es beheimatet ist? Die neuere Lehre ist wesentlich vorsichtiger. Zwar wird auch hier die Zweiteilung grundsätzlich akzeptiert; doch wird zugegeben, dass die Trennung „unscharf“ (Larroumet 1984: 47) und ihre Anwendung schwierig ist (Ghestin/Goubeau 1990: 68).

Die grundsätzliche Unterscheidung in Öffentliches Recht und Privatrecht wurde in Frankreich niemals in Frage gestellt; der Streit betraf vornehmlich Abgrenzungsfragen: das jeweilige Territorium sollte verteidigt oder auf Kosten des anderen ausgedehnt werden. Zum größten Teil schrumpft die Kontroverse auf einen Schulenstreit zwischen Privatrechtlern und Vertretern des Öffentlichen Rechts, wo jede Seite bemüht ist, die Verdienste der eigenen Disziplin herauszustreichen. Und da die Spaltung in Privates und Öffentliches Recht weithin das Rückgrat der Lehre an den Universitäten ist, bleibt ein Ausbrechen aus dem Teufelskreis schwierig: die Zusammenhänge zwischen den beiden Disziplinen, die ja getrennt gehalten werden, erscheinen bei ihrer Darstellung jeweils im Lichte des einen oder des anderen Standpunktes.

Schließlich bleibt noch zu bedenken, dass das Privatrecht, dem das Gebiet der Verträge, des freien Austauschs von Willenserklärungen und der zivilrechtlichen Haftung zuzuordnen ist, am ehesten für den wirtschaftlichen Liberalismus geeignet scheint, wohingegen das Öffentliche Recht als Verkörperung des staatlichen Zwanges gilt und den Verdacht von Dirigismus, wenn nicht gar von Sozialismus erweckt.

\section{Die beiden Bereiche des Internationalen Rechts}

Die nationale Aufteilung in privates und öffentliches Recht wiederholt sich auf der Ebene der internationalen Beziehungen. Im 19. Jh. wurden diese ausschließlich als zwischenstaatliche Beziehungen verstanden: nur die 
Staaten seien Völkerrechtssubjekte, was zur Folge hatte, dass sich die internationale Justiz auf Streitigkeiten zwischen verschiedenen Staaten beschränkte, wobei jeder Staat nur für seine Handlungen oder Unterlassungen gegenüber einem anderen Staat haftet. Demgegenüber regelt das Internationale Privatrecht die Beziehungen zwischen Privatleuten sowie die Beziehungen zwischen diesen und den Staaten, deren Staatsangehörigkeit sie nicht besitzen. Diese Aufspaltung des Rechts hat verschiedene Konsequenzen. Zum einen reproduziert das Internationale Privatrecht, da es als Teil des nationalen Rechts gilt, die territoriale Aufteilung der staatlichen Zuständigkeiten. Von daher ist es wenig geeignet für die Vertragsbeziehungen zwischen einem Staat und einem Unternehmen und es ist verständlich, dass schon mehrfach versucht worden ist, die „staatlichen Verträge“ einer adäquateren Regelung zu unterwerfen. ${ }^{1}$ Eine weitere Konsequenz der Trennung von öffentlichem und privatem Raum besteht darin, dass Individuen, die Opfer rechtswidrigen staatlichen Handelns geworden sind, kein anderer Rechtsbehelf zur Verfügung steht als eine Klage vor den Gerichten eben dieses Staates. Ohne jeden Zweifel hat die Praxis des 19. Jh. die Gewährleistung eines diplomatischen Schutzes entwickelt, der noch dazu durch internationale Rechtsmittel verstärkt worden ist. Allerdings hat der diplomatische Schutz und die Anwendung internationalen Rechts zwei wichtige Mängel. Weit davon entfernt, die Schranken zwischen dem Öffentlichen und dem Privaten niederzureißen, erhöht sie diese noch, da das diplomatische Vorgehen zur Folge hat, dass der von einem Einzelnen geltend gemachte Schaden auf die Ebene der zwischenstaatlichen Beziehungen gehoben wird. ${ }^{2}$ Der gravierendste Mangel besteht aber darin, dass die Praxis des diplomatischen Schutzes deutlich gemacht hat, dass sich dieser Schutz vor allem auf Eigentumsrechte bezieht, deren Verletzung einem fremden Staate zum Vorwurf gemacht wurde. Letztlich bestand dieser Schutz darin, dass Staaten, die noch einem quasi-kolonialen Einfluß unterliegen, die Rechtskriterien einer Handvoll Industriestaaten aufgezwungen wurden. Als die Enwicklungsländer nach dem 2. Weltkrieg auf der internationalen Bühne erschienen sind, hat das Recht des diplomatischen Schutzes daher auch eine heftige Kritik erfahren, heutzutage ist es praktisch diskreditiert (vgl. Castaneda 1961; Guha Roy 1961; Jiménez de Aréchaga 1978).

\section{Der Schutz der Grundrechte}

Die Unterscheidung des Internationalen Rechts in zwei Bereiche beginnt erst im frühen 19. Jh.; sie ist geprägt von dem seinerzeit herrschenden Rechtsposi-

1 Es existiert eine reichhaltige Literatur zu den „,contrats d'Etat“. Zur Begriffsbestimmung und zum Stand der Diskussion vgl. Rigaux (1989).

2 Die Rechtsprechung des Ständigen Internationalen Gerichtshofs ist dafür sehr erhellend. 
tivismus. Ursprünglich umfaßte das, was man „Völkerrecht“ nannte (ius gentium, law of nations, droit des gens), ohne jeden Unterschied sämtliche Aspekte der internationalen Beziehungen: sowohl die Beziehungen zwischen Staaten, als auch die Beziehungen zwischen dem Staat und den Angehörigen eines anderen Staates, sowie die Beziehungen der Privatleute untereinander. Nach dem 2. Weltkrieg stellte der internationale Schutz der Grundrechte einen ersten Versuch dar, eine überholte dogmatische Spaltung zu überwinden. Das wichtigste Beispiel dafür ist die Konvention zur Wahrung der Menschenrechte und der Grundfreiheiten, die am 4. November 1950 in Rom unterzeichnet worden ist, die sog. Europäische Menschenrechtskonvention. Seitdem kann der Staat für seine Handlungen vor einer Internationalen Gerichtsbarkeit zur Rechenschaft gezogen werden, und zwar für Sachverhalte, die eigentlich seiner eigenen Staatsgewalt unterliegen, sowohl im Hinblick auf die eigenen Staatsangehörigen als auch auf alle Ausländer, die in seinen Zuständigkeitsbereich fallen. Der Sicherheitsriegel des diplomatischen Schutzes ist zerbrochen, denn das Individuum, das Opfer einer Grundrechtsverletzung durch ein staatliches Organ ist, hat direkten Zugang zur internationalen Gerichtsbarkeit ${ }^{3}$, wo allerdings nur die Rechtsverletzung als solche festgestellt werden kann.

Schon vor der Verabschiedung der Konvention von 1950 und gleichzeitig mit der Annahme der Allgemeinen Menschenrechtserklärung durch die Vollversammlung der Vereinten Nationen (10.12.1948) hatten die Siegermächte des 2. Weltkriegs zwei internationale Militärgerichtshöfe eingesetzt. Der eine tagte in Nürnberg und fällte sein Urteil am 1. Oktober 1946, der andere tagte in Tokyo und sprach ein Urteil, das sehr lang war und dessen Bedeutung durch mehrere abweichende Meinungen relativiert wurde. ${ }^{5}$

3 Vor Verabschiedung des 11. Protokolls zur europäischen Menschenrechtskonvention vom 11.5.1994, das am 1.11.1998 in Kraft getreten ist, hatten Individuen nur Zugang zur Europäischen Menschenrechtskommission; von deren Entscheidung hing es dann ab, ob die Klage vor Gericht zugelassen wurde. Seit der Verschmelzung der beiden Organe sind Individual- oder Kollektivklagen vor dem Gerichtshof möglich (Art. 34 Europäische Menschenrechtskonvention, geändert durch Art. 1 des genannten Protokolls).

4 Der Europäische Gerichtshof für Menschenrechte bleibt in das Korsett der klassischen internationalen Gerichtsbarkeit eingezwängt: Er kann einen Staat nur wegen der Handlungsweise eines seiner Organe (also auch des Gesetzgebers) verurteilen; es bleibt ihm jedoch verwehrt, den staatlichen Behörden Anweisungen zu geben oder in der innerstaatlichen Rechtsordnung den Rechtsakt aufzuheben, der zur Klage geführt hat. Der in ihren Rechten verletzten Partei kann der Gerichtshof allein eine ,angemessene Befriedigung“ gemäß den Voraussetzungen nach Art. 41 (neu) der Konvention zusprechen.

5 Nach 7 Monate währenden Beratungen fällte das Internationale Militärgericht für den Fernen Osten ein Urteil, dessen Verlesung allein schon mehr als eine Woche in Anspruch nahm (4.-12. November 1948). Während die Verhandlungen gegen die großen Kriegsverbrecher vor dem Internationalen Militärgericht in Nürnberg ab 1947 von offizieller Seite veröffentlicht wurden (42 Bände in Französisch und Englisch), wurde das Tokyo-Urteil nur verspätet und privat veröffentlicht, vgl. Pritchard/Zaide (1987). 
Das Neue an den Militärgerichten von Nürnberg und Tokyo bestand darin, dass als international bezeichnete Gerichtshöfe über Individuen zu Gericht saßen, die wegen Kriegsverbrechen, Verbrechen gegen die Menschlichkeit und wegen eines Angriffskrieges angeklagt waren.

Zwischen dem Europäischen Gerichtshof für Menschenrechte und den beiden Internationalen Militärgerichtshöfen besteht eine perfekte Symmetrie: Einerseits wird der Staat aufgefordert, sich gegen die von einem Individuum erhobenen Anschuldigungen zu verteidigen, andererseits verwandeln sich Verletzungen des Kriegsrechts und der Gesetze der Menschlichkeit in Verbrechen nach internationalem Recht, wofür die entsprechenden Individuen zur Rechenschaft zu ziehen sind. In beiden Fällen hat eine internationale Gerichtsbarkeit das Mandat erhalten, um direkt zu urteilen, sei es über die von einem Individualkläger behauptete Rechtsverletzung des Staates, sei es über Verbrechen, die einer Person zur Last gelegt werden, die zwar Organ des jeweiligen Staates ist, aber jeglicher Funktionsimmunität entkleidet wird. Der Parallelismus ist um so bemerkenswerter, als die Völkerrechtsverbrechen die Verletzung von Grundrechten der Opfer betreffen.

Die aufgrund von Resolutionen des Sicherheitsrates der Vereinten Nationen veranlaßte Errichtung von Strafgerichten im Hinblick auf Ex-Jugoslawien und Ruanda ${ }^{6}$ sowie der Plan zur Schaffung eines Internationalen Strafgerichtshofes, der am 18. Juli 1998 in Rom angenommen wurde, verstärken die Auflösung der strengen Trennung zwischen dem öffentlichen Recht auf der einen Seite und internationalen Privat- und Strafrecht auf der anderen Seite.

\section{Kritik am Kriterium „Natur der Rechtssubjekte“}

Die neuere Entwicklung des internationalen Strafrechts und des Schutzes der Menschenrechte stößt auf begeisterte, allerdings nicht immer einsichti-

6 Statut des Internationalen Gerichtshofes für Ex-Jugoslawien, aufgrund der Resolution Nr. 827/1993 des Sicherheitsrates vom 25. Mai 1993; Statut des Internationalen Gerichtshofes für Ruanda, aufgrund der Resolution Nr. 955/1994 des Sicherheitsrates vom 8. November 1994.

7 Eine äußerst scharfsinnige Terminologie unterscheidet zwischen dem internationalen Strafrecht als Teil des Völkerrechts und dem Strafrecht mit internationalem Bezug, das zum innerstaatlichen Recht gehört. Das erste behandelt „Verbrechen gegen das Völkerrecht" und regelt deren Verfolgung. Das zweite legt das Ausmaß der strafrechtlichen Zuständigkeit der Staaten im Hinblick auf Straftaten fest, die Berührungspunkte mit einem anderen Land aufweisen. Dieses Rechtsgebiet steht mithin dem Internationalen Privatrecht sehr nahe. Die staatlichen Gerichte sind zuständig für die Kriegsverbrechen oder die Verbrechen gegen die Menschlichkeit, die in den jeweiligen einschlägigen Bestimmungen der nationalen Strafgesetze geregelt sind. Vor der Errichtung von Internationalen Strafgerichten waren die jeweiligen nationalen Gerichte allein zuständig. Diese Zuständigkeit haben sie auch weiterhin, da die Funktion der Internationalen Gerichtsbarkeit nur subsidiär ist und hinter einem staatlichen Gericht zurückstehen muß, falls dieses in gutem Glauben an seine Zuständigkeit tätig wird. 
ge Zustimmung der öffentlichen Meinung. Es werden jetzt „Subjekte“ einander gegenübergestellt, die früher in keiner Weise miteinander kommuniziert haben. In dieser Übergangsperiode des Völkerrechts ist es von Bedeutung den Stellenwert der Unternehmen, vor allem der transnationalen Unternehmen zu bestimmen.

Die traditionelle Rechtslehre weist dem Privatrecht einen abgegrenzten Bereich $\mathrm{zu}$, der durch die Rechtsnatur der Rechtssubjekte bestimmt ist. Im wesentlichen handelt es sich um Privatpersonen, inhaltlich geht es um Verträge und (zivilrechtliche) Haftung. Die Akteure des privaten Handels sind autonome Subjekte, die als gleich angesehen werden und die frei Verträge abschließen können, wobei unter dem Deckmantel einer utilitaristischen Ethik angenommen wird, dass jedes Subjekt im Einklang mit seinen eigenen Interessen handelt. Die Vertragsbeziehungen - Arbeit gegen Lohn, Wohnung gegen Bezahlung von Miete, normale Konsumgüter gegen einen „,vom Markt festgelegten“ Preis - besetzen einen klein gewordenen Raum, in dem der Staat und der Mechanismen des öffentlichen Rechts angeblich keinen Einfluß haben. Zweifellos ist das staatliche Recht langsam aber sicher in Beziehungen eingesickert, die bislang als privat galten. Aber die Grundsatzfrage, die von der klassischen Lehre eher verdunkelt als erhellt wird, ist, ob die privatrechtlichen Beziehungen, in dem geschrumpften Bereich, der ihnen noch verblieben ist, tatsächlich die sie konstituierenden Voraussetzungen tragen: den ,natürlichen“ Charakter eines generalisierten Marktes, zu dessen Funktionieren die staatlichen Institutionen und vor allem der dem Eigentum gewährte Schutz angeblich nichts beitragen. Ein Urteil des Obersten Gerichtshofs der Vereinigten Staaten, das ein Gesetz eines Staates für verfassungswidrig erklärt hatte, worin Arbeitgebern untersagt wurde, gewerkschaftlich organisierten Arbeitnehmern die Einstellung zu verweigern, enthält eine äußerst bezeichnende Begründung im Hinblick auf den Stellenwert der Vertragsfreiheit in einem marktwirtschaftlichen System.

„Und, da es selbstverständlich ist, dass, außer in dem Fall, dass alle Gegenstände in Gemeineigentum stehen, einige Leute mehr Eigentum als andere haben müssen, ist es naturgemäß unmöglich, die Vertragsfreiheit und das Recht auf Eigentum aufrechtzuerhalten, ohne gleichzeitig die Legitimität von ungleichen Vermögen zu bejahen, die sich als notwendige Folge der Ausübung dieser Rechte ergeben.“ (Coppage vs. Kansas, 236 US 1, 17, 1915)

Dieser Text enthält eine große Anzahl von nicht verifizierten Behauptungen, die allein durch das verbale Pathos Bestand haben: es ist selbstverständlich, sie müssen haben, es ist naturgemäß unmöglich, notwendige Folge. „Vertragsfreiheit und Eigentumsrecht" sind die Grundwerte des Lebens in einer Gesellschaft, denen sich alle anderen Elemente unterordnen müssen. Die Schuldenkrise der Länder der „Dritten Welt“ ist ein typisches Beispiel einer derartigen Unterordnung der Grundrechte unter die (als naturgegeben unterstellten) Gesetze des Marktes. Die Pflicht, den Schulden- 
dienst sicherzustellen und die Schuld zurückzuzahlen, wird erst gar nicht zur Diskussion gestellt. Alle Vereinbarungen werden im Hinblick auf die Interessen der Gläubiger getroffen: die jeweilige Politik der Weltbank und des Internationalen Währungsfonds sind voreingenommen zugunsten der Reichen. Was man „strukturelle Anpassung“ nennt, berücksichtigt nicht die Auswirkungen der den Schuldnerländern aufgezwungenen Maßnahmen auf das Wohlbefinden, die Gesundheit, die Ausbildung der Bevölkerung, die gezwungen ist, die von den Regierenden eingegangenen Schulden zu bezahlen.

Es muß nochmals betont werden: Selbst in den Bereichen, wo das Privatrecht noch immer herrscht, ist die Vorstellung, wonach es sich dabei um ein der staatlichen Gewalt entzogenes Rechtsgebiet handelt, völlig realitätsblind. Es sind die staatlichen Institutionen, die das Eigentumsrecht garantieren - auch durch die einschlägigen Verfassungsbestimmungen ${ }^{8}$, die seine erbrechtliche Übertragung regeln, die den ,rechtmäßig geschlossenen Verträgen“ (Art. 1134 Code Civil) Gesetzeskraft verleihen und Zwangsvollstreckung ermöglichen, die die Errichtung von Kapitalgesellschaften erlauben und ihre Ausdehnung jenseits der nationalen Grenzen tolerieren.

Weit davon entfernt, eine bloß technische Unterscheidung zu sein, stellt die Einteilung in Öffentliches und Privates Recht einen Ort der Macht einem Bereich der Freiheit und der Nicht-Macht gegenüber: Macht gegen Recht, Macht gegen Privatautonomie, öffentliche Autorität gegen freies Austarieren der Interessen. Die Unterscheidung ist rein ideologisch: das Private ist friedlich, unschädlich, wohltuend; das Öffentliche ist bedrohlich und gefährlich. Daher stellt sich die Frage, ob die Gegenüberstellung von Privatem und Öffentlichem nicht gerade aufgrund der Macht, die die Inhaber des „Privat"eigentums angehäuft haben, überholt ist.

Schon seit langer Zeit weiß man, dass Eigentum Macht verleiht und zwar sowohl den Individuen als auch den Unternehmen. Zahlreiche Beziehungen zwischen formal Gleichen beinhalten eine Machtstruktur. Die Beziehungen zwischen Wohnungseigentümer und Mieter, zwischen Darlehensgeber und Darlehensnehmer, zwischen Arbeitgeber und Beschäftigten sind geprägt von der Überlegenheit der Ersteren gegenüber den Letzteren, so dass diejenige der beiden Parteien, deren existentielle Bedürfnisse den Abschluß des

8 Die Kommentatoren des Entwurfs der Erklärung der Menschen- und Bürgerrechte und des ersten Entwurfs einer französischen Verfassung stimmen darin überein, dass die erste Zielsetzung des neuen Regimes die Verteidigung des Eigentums ist. Vgl. u.a. die Reden von Sieyès vom 20. und 21. Juli 1789 und von Barnave vom 11. August 1791, (Orateurs... 1989: 1014, 44-45). Beide Redner rechtfertigen das Klassenwahlrecht mit der notwendigen Verbindung von Eigentum und der Ausübung politischer Rechte. Nach Sieyès sind „citoyens actifs“ (aktive Bürger), zu denen nicht die Frauen, die Kinder und die Fremden gehören, nur diejenigen, die „,an dem öffentlichen Gemeinwesen“ (durch ihr Eigentum) mitwirken und die damit allein ,die wahren Aktionäre des großen Gesellschaftsunternehmens sind“ (ebd.: 1014). 
Vertrages erfordern, keineswegs frei in der Entscheidung ist, ob sie die gestellten Bedingungen akzeptieren will oder nicht.

Diese Gleichheitsillusion hat den Blick fürs Wesentliche verstellt; mit der Unterscheidung in Öffentliches Recht und Privatrecht werden alle ,privaten" Akteure als gleich behandelt, vom brasilianischen oder philippinischen Landarbeiter bis hin zum mächtigsten transnationalen Unternehmen. Folglich kann das Kriterium der Macht nicht den Inhabern staatlicher Autorität vorbehalten bleiben; zahlreiche „private“ Rechtspersonen üben eine beträchtliche Macht aus, die sogar weit über die der meisten öffentlichen Hoheitsträger hinausgeht, und die um so hinterhältiger ist, als sie nicht im Gewande der Macht daherkommt. Der kleine Staatsbeamte läßt sich von einem einflußreichen Unternehmer einschüchtern, in seinen Handlungsmöglichkeiten einschränken oder auch bestechen. Und noch schlimmer wird es, wenn mafiöse Machtstrukturen berücksichtigt werden, deren regelmäßige Gewaltausübung und deren Wirksamkeit denen der staatlichen Hoheitsträger bei weitem überlegen ist.

Weit davon entfernt naturgegeben zu sein, sind die ökonomischen Ungleichheiten die Folgen des Systems von Eigentum und Verträgen, d.h. die Folgen einer Rechtsordnung, die durch die Zwangsgewalt des Staates aufrechterhalten wird. Der Markt ist gerade kein natürlicher Ort des Tausches, der auch ohne die staatliche Gewährleistung sowohl der zwingenden Wirkung der Verträge als auch der erbrechtlichen Weitergabe des Eigentums funktionieren könnte (vgl. dazu Unger 1987, Waldron 1990, Scheiber 197, Ward 1998).

\section{Der Konflikt zwischen Eigentumsrecht und Meinungsfreiheit}

Die amerikanische Rechtsprechung bietet ein eklatantes Beispiel vom künstlichen Charakter der traditionellen Unterscheidung von Öffentlichem Recht und Privatrecht. In dem betreffenden Fall ging es um den Konflikt zwischen dem Recht des Eigentümers bestimmter Örtlichkeiten, deren Zutritt er einer unbestimmten Anzahl von Personen gestattet hat, und der Meinungsfreiheit eben dieser Personen. Kann dieser Eigentümer, indem er die Bedingungen festsetzt, unter denen das Publikum Zutritt zu der betreffenden Örtlichkeit hat, dort die Ausübung der Meinungsfreiheit einschränken? Das Problem liegt darin, dass der Raum als Eigentum privat ist, aber gleichzeitig öffentlich, da der freie Zugang gestattet ist. Es ist nicht nötig, auf die Lösungen des Höchsten Gerichts der Vereinigten Staaten einzugehen, das eher das Eigentumsrecht als die Meinungsfreiheit in den Vordergrund stellte. Die grundsätzliche Bedeutung wird anhand einiger Beispiele deutlich. Darf in einer Siedlung, die eine Firma zum Zwecke der Unterbringung von Arbeitern gebaut hat, die Verbreitung von Broschüren religiöser Eiferer eingeschränkt werden? Darf ein Unternehmen seinen Mitarbeitern untersagen, Streikposten auf dem Gelände aufzustellen, wo sie ihre ge- 
schäftlichen Aktivitäten betreibt? Ist es rechtens, die Verteilung von Broschüren in einem Einkaufszentrum oder in einem Flughafenterminal zu untersagen? Darf ein Unternehmen seinen Arbeitnehmern verbieten, sich auf einem an das Firmengelände angrenzenden Parkplatz zu versammeln? Diese (tatsächlich aufgetretenen) Konflikte verdeutlichen, wie wenig die Unterscheidung von Öffentlichem Recht und Privatrecht noch praktikabel ist: Wenn das jeweilige Gelände lediglich privat wäre, so könnte der Eigentümer ohne jede Einschränkung die Ausübung aller verfassungsrechtlich garantierten Freiheiten begrenzen; diese Freiheiten gelten nur in dem Maße, wie das Gelände öffentlich ist.

Ein zweites Beispiel bietet die Aneignung der Massenkommunikationsmittel. Das war schon immer der Fall für die gedruckte Presse, die Entwicklung hat sich aber mit der Privatisierung von Radio und Fernsehen noch beschleunigt. Außerdem ist hier der Staat in erheblich größerem Maße mit einbezogen, denn er vergibt die Frequenzen und übt eine viel stärkere Kontrolle über den audiovisuellen Bereich aus als über die gedruckte Presse. Die im Hinblick auf die Vereinigten Staaten angestellte Überlegung, dass die „Verteilung der Redemacht weder natürlich noch politisch ist“ (Sullivan 1992: 105), gilt ebenso für Europa. Die Fähigkeit sich auszudrücken, ist, genauso wie das Eigentum, von einem Recht zu einem Machtmittel umgeschlagen. Ein amerikanischer Autor hat die Bemerkung gemacht, dass der amerikanische Fernsehsender CBS ,weder ein staatlicher Akteur noch ein einfacher Bürger ist, sondern vielmehr etwas von beiden hat. ... CBS ist folglich eine Mischung aus Öffentlichem und Privatem“ (Fiss 1986: 1414).

Die Macht, die das Privateigentum verleiht, ist um so bedeutsamer, als die Produktionsmittel konzentriert sind und das Eigentumsrecht mittlerweile auch die Urheberrechte mit einschließt, das industrielle Know how, das etwa für Schürfarbeiten und die Ausbeutung von Erdöllagern so wichtig ist. Es erscheint mir daher bewiesen zu sein, dass der Konzern Elf-Aquitaine eine Macht darstellt, und zwar eine um so unwiderstehlichere Macht, als sie gegenüber den afrikanischen Staaten in Form einer - wie es die Anklageschrift formuliert - doppelten Einmischung von außen ausgeübt wird: Zum einen sind diese Staaten der Einmischung eines fremden Unternehmens ausgesetzt, das zahlreiche Aspekte der nationalen Wirtschaft determiniert, zum andern unterliegen sie der Einmischung des Staates, dem dieses Unternehmen angehört; und dessen Politik gegenüber Afrika ist alles andere als selbstlos, sie unterstützt vielmehr die Interessen seiner Unternehmen. 


\section{Müssen die transnationalen Unternehmen die Grundrechte achten?}

Das vorhanden Recht

Was hier wieder ins Gewicht fällt ist die Trennung von Öffentlichem Recht und Privatrecht. Die internationalen Instrumente zum Schutz der Menschenrechte sind in einem Gefühl des Mißtrauens gegenüber den Staaten konzipiert worden. Auf die gleiche Art und Weise, wie die liberalen Verfassungen eine Schutzmauer um die Freiheiten des Individuums herum errichtet haben, haben die internationalen Verträge zum Schutz der Menschenrechte ein zusätzliches Stockwerk in die Zitadelle eingebaut und haben sie mit Wachttürmen versehen, von wo aus der internationale Beobachter den Staat tadeln kann, der seinen Verpflichtungen nicht nachkommt. Andererseits, und dies entgegen allen allzu häufig vorgetragenen und leichtfertigen Behauptungen, erlegen weder die nationalen Verfassungen noch die Verträge zum Schutze der Grundrechte den Individuen irgendwelche Verpflichtungen auf. Was man manchmal die Drittwirkung dieser Bestimmungen nennt, ist nichts anderes als die Schlußfolgerung, die der Staat in seinem staatlichen Recht im Hinblick auf seine eigenen Verpflichtungen zieht, sei es dass ein nationales Gesetz den Privatleuten ähnliche Pflichten auferlegt (beispielsweise betrifft die Pflicht, die Privatsphäre zu achten, sowohl Privatpersonen als auch die Staatsorgane), sei es dass die Justiz die Normen des nationalen Rechts, die auf die Beziehungen zwischen Privatpersonen anzuwenden, sind in Zusammenhang mit den vom Staat garantierten Grundrechten interpretiert (Ausstrahlungswirkung). So kann ein Journalist oder ein Presseorgan, das wegen Verleumdung verfolgt wird, seine Meinungsfreiheit gegenüber dem Kläger geltend machen.

Vor dem Europäischen Gerichtshof für Menschenrechte kann ein Unternehmen nur als Kläger auftreten; es kommt durchaus vor, dass der Gerichtshof auf den Antrag einer juristischen Person hin einen Staat wegen Verletzung eines Grundrechts (insbesondere des Rechts auf Eigentum) oder einer Grundfreiheit verurteilt, weil nämlich das Unternehmen als eine Person angesehen wird, die nach Art. 1 der Konvention in den Zuständigkeitsbereich des betreffenden Staates fällt. Auf der anderen Seite unterliegt ein Unternehmen ebensowenig wie irgend ein anderes ,,privates Rechtssubjekt" der internationalen Gerichtsbarkeit wegen der Verletzung von Grundrechten, die es gegenüber einem anderen Rechtssubjekt begangen hätte.

Damit ein Unternehmen gezwungen ist, die Grundrechte zu achten, muß eine derartige Verpflichtung aufgrund des nationalen Rechts des Staates bestehen, dem es angehört oder auf dessen Territorium es tätig wird. Insbesondere muß ein derartiges Unternehmen die dort geltenden arbeitsrechtlichen Bestimmungen oder die Vorschriften zum Umweltschutz einhalten. Es gibt allerdings eine Reihe von Grundrechten, denen der nationale Ge- 
setzgeber keine zwingende Wirkung in den Beziehungen der privaten Rechtssubjekte untereinander beigelegt hat - so etwa dem Recht auf Arbeit, dem Recht auf Gesundheit oder dem Recht auf eine nicht verseuchte Umwelt. Meistens handelt es sich dabei um Leistungs- bzw. Teilhaberechte, die der Staat selbst nicht mit der gleichen Intensität gewährleisten kann, wie er sich jeder rechtswidrigen Handlungsweise als Öffentliche Gewalt enthalten muß (Abwehrrechte).

Selbst was die Grundrechte betrifft, die sich in der nationalen Rechtsordnung wiederfinden und zwingend in den Beziehungen zwischen Privaten gelten, können sich die Multis leicht ihrer Verpflichtung entziehen, sie einzuhalten. Die Aufteilung der staatlichen Zuständigkeiten zwischen politisch getrennten Territorien begünstigt eine faktische Immunität der Unternehmen. Einerseits ist der Staat, in dem der Multi in problematischer Weise tätig wird, häufig ein unterentwickeltes Land, das den ausländischen Investoren attraktive Bedingungen einräumt, woraus sich ein entsprechend niedrigeres Schutzniveau für die Arbeitnehmer ergibt. Andrerseits ist der Staat, dem das Unternehmen angehört, kaum darum bemüht, seine Unternehmen zur Achtung der Bestimmungen anderer Staaten anzuhalten, die dem (oft unzulänglichen) Schutz der Bevölkerung dienen sollen. Die staatliche Inaktivität auf diesem Gebiet wird mit einem allzu bequemen Argument begründet: der Achtung der Souveränität des anderen Staates.

\section{Das noch zu schaffende Recht}

Im Unterschied $\mathrm{zu}$ den Individuen, staatlichen Organen oder einfachen Bürgern, die neuerdings vor einem Internationalen Strafgerichtshof wegen der schlimmsten Verbrechen nach internationalem Recht angeklagt werden können, müssen die Kapitalgesellschaften und insbesondere die transnationalen Unternehmen sich nur vor staatlichen Gerichten verantworten, die wenig Neigung zu ihrer Verurteilung zeigen oder sich durch die Diskrepanz zwischen der staatlichen Ohnmacht und der Macht eines derartigen Unternehmens daran gehindert sehen.

Damit die transnationalen Firmen wegen ihrer Handlungen zur Rechenschaft gezogen werden können, müßten zwei Voraussetzungen erfüllt sein. Zunächst müßten den Multis, entsprechend dem Umfang ihrer Macht, auch eine Reihe von Verpflichtungen auferlegt werden. Die Haltung, die von den internationalen Strafgerichten im Hinblick auf das Unterlassungsdelikt eingenommen wird, ist insoweit aufschlußreich. Ein Vorgesetzter, der Kenntnis hat von Verbrechen, die seine Untergebenen begehen, ist der Beihilfe an diesen Verbrechen schuldig, wenn er nicht alles getan hat, was er hätte tun können - und müssen -, um diese Verbrechen zu verhindern. Die Haftung der transnationalen Unternehmen für Schäden, die sie an Personen, an Gütern und an der Umwelt verursachen, muß an der Elle ihrer Machtpo- 
sition gemessen werden. Die Parallele zum Völkerstrafrecht kann aber noch weiter verfolgt werden. Selbst wenn das staatliche Recht ein Kriegsverbrechen oder ein Verbrechen gegen die Menschlichkeit erlaubt, zuläßt oder anordnet, selbst wenn ein Untergebener den Befehl erhalten hat, ein derartiges Verbrechen zu begehen, wird dadurch seine Verantwortung im Hinblick auf das Internationale Strafrecht nicht berührt. Eine ähnliche Überlegung wäre auch bezüglich des geltenden Rechts in den unterentwickelten Ländern anzustellen, die von den dort tätigen Multis abhängig sind. Die zweite Voraussetzung ist die Existenz einer internationalen Gerichtsbarkeit, die in der Lage wäre, über die Haftung der Multis zu befinden (ob über die strafrechtliche oder nur über die zivilrechtliche Haftung, kann hier dahingestellt bleiben). Der Plan zur Errichtung eines Ständigen Internationalen Strafgerichtshofes macht die Lücke in der internationalen Ordnung schmerzhaft deutlich; er sollte auch Anlaß zur Schaffung einer internationalen Gerichtsbarkeit im Hinblick auf die Haftung der transnationalen Unternehmen sein. Solange jedoch keine derartigen Ergebnisse vorliegen, muß das Ständige Tribunal der Völker eine Aufgabe wahrnehmen, die bislang kein anderes Organ - weder nach staatlichem noch nach internationalem Recht - wahrgenommen hat.

\section{Die internationale Verantwortlichkeit des Staates für ,seine“ Unternehmen}

Das Attribut „transnational“ oder „,multinational“, unter dem ein „privates Unternehmen“ daherkommt, sollte nicht zu Fehlschlüssen über die Zugehörigkeit eines derartigen Unternehmens verleiten. Man könnte sagen, dass sie nirgendwo hingehören, denn die Delokalisierung, die von den Unternehmen durchgeführt worden ist, und die Aufteilung ihrer Aktivitäten auf mehrere unterschiedliche Rechtssubjekte haben in weitem Umfang jede Zugehörigkeit zu einem einzelnen Staat aufgehoben. Das Land der Muttergesellschaft, wo die wesentlichen Entscheidungen getroffen werden, bleibt jedoch ein Anknüpfungspunkt, denn es ist dieses Land, wo der Konzern bei Schwierigkeiten Unterstützung suchen wird. Gleichgültig ob nun der Konzern als Ganzer betrachtet wird oder jedes der beteiligten Unternehmen einzeln, es handelt sich stets, im Rahmen des gegenwärtigen Rechtssystems, um Privatrechtssubjekte, die grundsätzlich der alleinigen Zuständigkeit des (jeweiligen) Staates unterliegen.

Im internationalen Recht gibt es analog zur zivilrechtlichen Haftung innerhalb des nationalen Rechts eine internationale Haftung des Staates. Es sind dies, nebenbei gesagt, die Bestimmungen, die auf den Regelungskomplex des diplomatischen Schutzes angewandt wurden. Allerdings trägt ein Staat Verantwortung nur gegenüber einem anderen Staat oder gegenüber einem 
anderen Subjekt des internationalen Rechts, wie z.B. oder UNO oder der Europäischen Union. Es gibt aber keine internationale Haftung des Staates gegenüber einem Volk oder einer Gruppe von Individuen; kein internationales Gericht könnte, nach dem derzeitigen Stand des internationalen Rechts, mit einer derartigen Beschwerde befaßt werden.

Es gilt noch ein anderes Hindernis zu überwinden: Die internationale Haftung eines Staates bezieht sich grundsätzlich nur auf die Handlungen seiner Organe. Der Staat ist nach dem derzeitig geltendem Recht international nicht für die Handlungen einfacher Bürger oder Unternehmen verantwortlich, es sei denn man kann beweisen, dass diese Person oder dieses Unternehmen im Auftrag des Staates gehandelt hat oder dass ein Staatsorgan an den rechtswidrigen Handlungen des privaten Rechtssubjekts beteiligt war. Aber auch dann tritt die Haftung des Staates nur im Verhältnis zu demjenigen Staat ein, der Opfer rechtswidriger Umtriebe geworden ist; eine internationale Gerichtsbarkeit könnte nur auf Antrag des verletzten Staates mit einer Haftungsklage befaßt werden, und dies auch nur dann, wenn beide Staaten eine Vereinbarung über eine gerichtliche Regelung des Streitfalles getroffen haben.

\section{Die subsidiäre Rolle des Ständigen Tribunals der Völker}

Angesichts dieser Lücken im geltenden internationalen Recht, sowohl auf der Ebene des Normensystems als auch angesichts des Fehlens einer den Völkern zur Verfügung stehenden Gerichtsbarkeit, obliegt es dem Ständigen Tribunal der Völker die Aufgabe eines Stellvertreters wahrzunehmen. Was zunächst die auf transnationale Unternehmen anwendbaren Normen betrifft, so müssen sie einem Mindeststandard in Sachen Grundrechtsachtung unterworfen werden. Dies schließt insbesondere die Rechte der Arbeitnehmer ein sowie die Rechte der Subunternehmer, den Umweltschutz, die Verpflichtung, sich jeglicher Einmischung in die inneren Angelegenheiten und die Regierungsgeschäfte des Gastlandes zu enthalten. Die diversen, von den transnationalen Gesellschaften erarbeiteten Verhaltenscodices (vgl. Rigaux 1989), die allerdings nur auf dem Papier stehen, könnten zu diesem Zwecke herangezogen werden. Die von den Multis in den wirtschaftlich abhängig gebliebenen Ländern ausgeübte Macht zieht Verpflichtungen nach sich, denen sich diese Unternehmen nicht unter Berufung darauf entziehen können, sie würden sich an die geltenden Gesetze halten, die häufig nur unzureichend umgesetzt werden.

Angesichts des Fehlens jeglicher Gerichtsbarkeit - sowohl staatlicher als überstaatlicher Art - vor welchen sich die Multis gegen die von den Völkern bzw. den sie repräsentierenden Organisationen erhobenen Vorwürfe verteidigen müßten, ist es Sache des Ständigen Tribunals der Völker hier eine Vertretungsfunktion auszuüben. 


\section{Schlußfolgerung}

Es obliegt den Mitgliedern des Gerichts darüber zu befinden, ob die Anklagepunkte gegen die Gesellschaft Elf-Aquitaine und gegen die französische Regierung durch die Tatsachen betätigt werden. Ziel diese Berichtes war es, an der Einschätzung der Tatsachen mitzuwirken.

Ein derart großes Unternehmen wie Elf-Aquitaine kann nicht als eine Rechtsperson aufgefaßt werden, die allein den Bestimmungen des Vertrags- und des Deliktrechts unterläge. Es handelt sich hierbei um einen Machtträger, dessen Verhalten bestimmten Anforderungen entsprechen muß, wie der Achtung der Grundrechte der Einzelpersonen und der menschlichen Gemeinwesen sowie des Völkerrechts. Nach dem gegenwärtigen Rechtszustand gibt es kein staatliches oder überstaatliches - Gericht, vor dem sich ein solches Unternehmen für die ihm zur Last gelegten Taten verantworten müßte.

Dem beklagten Staat kommt eine noch weiter gehende Immunität zugute: Er könnte nur auf Antrag eines anderen Staates vor eine internationale Gerichtsbarkeit zitiert werden. Trotzdem: Falls bewiesen wird, dass die französische Regierung an den rechtswidrigen Handlungen beteiligt war, deren Elf-Aquitaine angeklagt ist, dann müßte sie zum Ersatz des Schadens verurteilt werden, den Elf den afrikanischen Völkern zugefügt hat, in deren Namen die Beschwerde erhoben worden ist.

\section{Übersetzung aus dem Französischen von Bertram Michel}

\section{Literatur}

Carbonnier, Jean (1969): Droit civil, 8e éd., Paris.

Castaneda, Jorge (1961): The underdeveloped nations and the development of international law, International Organizations 15, S. 36-48.

Fiss, Owen M. (1986): Free Speech and Social Structure, Iowa Law Review 31, 1405-1425.

Ghestin, Jacques; Goubeau, Gilles (1990): Traité de droit civil, 3é éd., Paris.

Guha Roy, S.N. (1961): Is the law of responsibility of states for injuries to aliens a part of universal international law?, in: The American Journal of International Law 55, S.863-891.

Jiménez de Aréchanga, E. (1978): State Responsibility of Foreign-Owned Property, in: New York University Journal of International Law and Politics 11, S.79ff.

Larroumet, Christian (1984): Droit civil, Bd. 1, Paris.

Orateurs de la Révolution française, Bd. 1, Les Constitutions (Bibl. De la Pléiade), 1989.

Pritchard, R. John; Zaide, Sonia Magbanua (Hrsg.) (1987): The Tokyo War Crimes Trial, New York und London.

Rigaux, Francois (1989): Les situations juridiques dans un système de relativité générale, in: Recueil des cours de l'Académie de droit international, Bd. 213 (1989-1).

Scheiber, Harry N. (1997): Private Rights and Public Power: American Law, Capitalism and the Republican Polity in Nineteenth Century America, in: The Yale Law Journal 107, 823-861.

Sullivan, Kathleen M. (1992): The Justices of Rules and Standards, in: Harvard Law Review 106, 22-123.

Unger, Roberto Mangabeira (1987): False Necessity, Anit-Necessitarian Social Theory in the Service of Radical Democracy, Cambridge University Press.

Waldron, Jeremy (1990): Criticizing the Economic Analysis of Law, in: The Yale Law Journal 99, 1441-1471.

Ward, Ian (1998): Law, Liberty, and Literature, in: Anglo-American Law Review 27, 188-220. 